\title{
AIAA 99-2841
}

Space Electrodynamic Tether Propulsion Technology: System Considerations \& Future Plans

Brian E. Gilchrist, ${ }^{1}$ Les Johnson, ${ }^{2}$ and Sven G. Bilén ${ }^{1}$ ${ }^{1}$ Space Physics Research Laboratory, University of Michigan, Ann Arbor, Michigan 48109

${ }^{2}$ Marshall Space Flight Center, Huntsville, Alabama 35812

\section{5th AIAA/ASME/SAE/ASEE Joint Propulsion Conference and Exhibit 20-24 June 1999 Los Angeles, California}




\title{
SPACE ELECTRODYNAMIC TETHER PROPULSION TECHNOLOGY: SYSTEM CONSIDERATIONS AND FUTURE PLANS
}

\author{
Brian E. Gilchrist*, Les Johnson†, Sven G. Bilénł
}

\begin{abstract}
Space electrodynamic tethers offer the opportunity for in-space "propellantless" propulsion around planets with a magnetic field and ionosphere. This propulsion is accomplished by converting the magnetic force on the tether current into propulsive or drag thrust to either increase or decrease the orbital energy of the spacecraft system. To validate electrodynamic-tether thrusting, we must be able to demonstrate sustainable currents and effective methods of collecting and emitting electron current under varying ionospheric conditions. To date, significant tether current flow $(\sim 1 \Lambda)$ to/from the tether's end collectors in the Earth's ionosphere has been demonstrated as part of NASA's TSS-1R mission in 1996, though the maximum possible current was not tested. Here, we review system-level. issues associated with effective electrodynamic-tether operation, which include ionospheric and motional-EMF variability as well as details of the tether "end". contacts under varying ionospheric conditions. We also survey potential future near-Earth applications of electrodynamic tethers as currently conceived. Applications ranging from orbit transfer of payloads, orbit maintenance, and end-of-mission deorbiting will be described. The NASA ProSEDS mission, an important first step to understanding electrodynamic-tether capabilities; is discussed.
\end{abstract}

\section{1-Introduction}

Space electrodynamic (ED) tethers offer the opportunity for in-space "propellantless" propulsion and power generation around planets with a magnetic field and an ionosphere (e.g., Earth and Jupiter). In general, moving a conductor across a magnetic field generates an electromotive force (EMF) to drive current through the conductor if a means to "close the circuit" is available. For example, using gravity gradient stabilized space tethers around Earth, it is possible to have kilometer-scale structures that move across the geomagnetic field at rapid velocities generating $50-250 \mathrm{~V} / \mathrm{km}$ EMF in an eastwardmoving system at a mid- to low-latitude orbit inclination [1]. Current flow through the tether is enabled by collecting electrons from the ionosphere at or along one end of the tether and, at the opposite end, either injecting electrons back into the ionosphere or collecting ions [2].

If the current flows in response to this motional EMF it can be utilized as a source of electrical power that is drawn from the orbital energy of the spacecraft. Thus, power generation comes at the expense of lowering the orbit of the spacecraft. However, this also means that the ED tether can provide a means for generating :"propellantless" propulsive forces by converting a magnetic force on a current along a tether into a thrust for the spacecraft $[1,3,4]$. In general, the

\footnotetext{
* Associatc Professor, Electrical Enginecring and Space Sciences, AIAA senior member

${ }^{\dagger}$ NASA Marshall Space Flight Center. AIAA scnior member

‡ Research Fcllow, Radiation Laboratory and Space Physics Research Laboratory, AIAA Mcmber

Copyright $\odot 1999$ by Brian E. Gilchrist. Published by the American Institute of Aeronautics and Astronautics, Inc. with permission.
}

thrust can be orbit-raising, orbit-lowering, or out-ofplane depending on the direction of current flow and the tether orientation relative to the magnetic field. For orbit-raising situations an alternate energy source, such as from solar cells, must overcome the motional EMF and reverse the current direction.

Below, we discuss results to date that give us confidence the technology we present in Section 2 will be practical. In Section 3 we discuss use of distributed electron collection along the tether rather than at just the tether end point. Section 4 describes the next step in tether technology development, the ProSEDS ED tether mission. Section 5 describes ED tether applications particularly useful for low Earth orbit (LEO). We then recommend the next logical steps for ED tether technology development (beyond ProSEDS) in Section 6.

\section{Results To Date}

Significant tether current flow $(\sim 1 \mathrm{~A})$ to/from an electrodynamic tether in the Earth's ionosphere has been demonstrated previously near $300-\mathrm{km}$ altitude as part of NASA's TSS-1R mission in 1996 [5], though the system's maximum possible current was not reached, Analysis of the measured TSS-1R tether currents indicates that electron current collection levels to its upper spherical collector (which was large with respect to a Debye length) exceeded those predicted for stationary, collisionless magnetoplasmas by two to three times [6]. The TSS-1R data do not, however, appear to point to a dependence of current on voltage greatly different from that of Parker-Murphy (PM) for higher voltages." Even though, for example, a TSS $-1 \mathrm{R}$ current of $0.5 \mathrm{~A}$ at 
$350 \mathrm{~V}$ bias may surpass PM model estimates, it could still imply a voltage of roughly $35 \mathrm{kV}$ to reach 5. A for the same plasma parameters requiring over $175 \mathrm{~kW}$ for a thrust of $0.7 \mathrm{~N}$ and a $10-\mathrm{km}-\mathrm{long}$ tether!

At the other end of the tether, electron emission is necessary to achieve the highest possible currents given the low mobility of ions. The use of a hollowcathode plasma contactor (HCPC) is the preferred method for emitting electrons because of its expected low sheath potential $(\sim 20-100 \mathrm{~V})$. A low sheath potential is important since it must be overcome by the source driving current through the tether, which decreases system efficiency. HCPCs, however, require an expendable gas supply. Alternative structures based on field emitter array cathodes (FEACs) are being studied since they appear to allow for a reasonably low sheath potential, but do not require any expendables [7]. This is discussed further in Section 6 below.

\section{3-Bare-Tether Electron Collection}

An alternative geometry, using so-called "bare" tether conductors/collectors, has been proposed by Sanmartin et al. [8]. It is expected that the bare tether will provide a more effective collector of ionospheric electrons (per unit area) than, say, a large sphere (such as the TSS-1R satellite) at equal bias. This is because the tether's small cross dimension allows electron collection in the orbital-motion-limited (OML) regime, which should give the highest possible current density. A bare-tether design represents a breakthrough that makes short-tether electrodynamic reboost with moderate power requirements practical:

The ED tether itself, if left uninsulated over a portion, will function as its own very efficient anode. The tether is biased positively with respect to the plasma along some or all of its length. This is due to the fact that the positively biased, uninsulated part of the tether then collects electrons from the plasma.

The following features argue in favor of the baretether concept $[9,10]$ :

1. The small cross-sectional dimension of the tether makes it a much more effective collector of electrons from the space plasma (per unit area) than is a large sphere (such as the TSS $-1 \mathrm{R}$ satellite) at equal bias. This is because the small cross dimension of the tether allows its current collection to take place in the $\mathrm{OML}$ regime, which gives the highest possible current density.
2. The large current-collection area is distributed along the tether itself, eliminating the need for a resource-using plasma contactor or a large, massive, and/or high-drag sphere at the electron collecting end of the tether. This substantially reduces the center-of-gravity shift in both cases and reduces the cost and complexity in the case of the active contactor.

3. The system is self adjusting to changes in electron density. This is accomplished by a natural expansion of the portion of the tether that is biased positively relative to the ionosphere whenever the density drops (this is shown later in Fig. 2).

Charged-particle collection is governed by the stronger gradients associated with the smaller dimensions and is thus a $2 \mathrm{D}$ process, the length being irrelevant to the density of current collected. For a radius small compared to both Debye length and electron gyroradius, there are neither space-charge nor magnetic-guiding effects, and we are in the $\mathrm{OML}$ regime. In this regime; the current takes the largest possible value for the given geometry and bias. Better still, it turns out that in cylindrical geometry the OML regime holds for radius-to-Debye length ratios even of order unity [17]. Hence, a cylinder of $5-\mathrm{mm}$ radius (about one Debye length and small compared with the electron gyroradius) works as an electron collector in the OML regime Fig. 1 shows the current collection efficiency of the bare tether compared to a sphere of equal area using TSS-1R data:

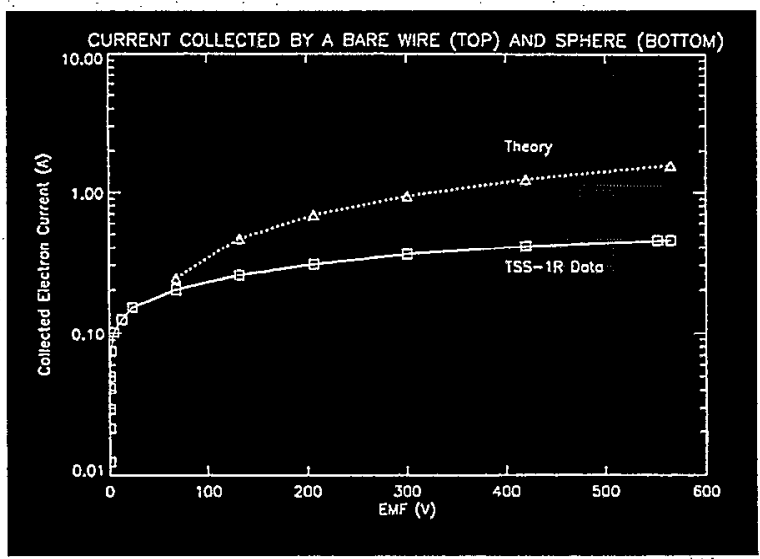

Figure 1-Current collection efficiency of the bare tether compared to a sphere of equal area.

For an orbiting, current-carrying tether the bias will actually vary along the tether because of both the motional electric field and the ohmic voltage drop. The electron current to the tether will thus vary with height. Along the uninsulated part of the tether, the tether current will decrease with decreasing altitude, until the point is reached at which the tether is at zero 
bias with respect to the plasma (or the end of the tether is reached). Assuming there is a point of zero bias on the tether, then below that point the tether is biased negative and an ion current will be collected. This ion current is much smaller because of the high ratio of ion mass to electron mass and decreases the average tether current somewhat.

The bias required to collect a given OML current varies as the inverse square of the collecting area, making it possible to reduce the required bias substantially by modestly increasing the collecting area. Since the current collected by an electroncollecting length $\mathrm{L}_{\mathrm{B}}$ grows roughly as $\left(\mathrm{L}_{\mathrm{B}}\right)^{1.5}$, the tether can automatically accommodate drops in density by increasing the length of the collecting segment, shifting the zero-bias point downward. (Fig. 2) This ability to maintain thrust levels with low electron densities makes night-time boost possible.

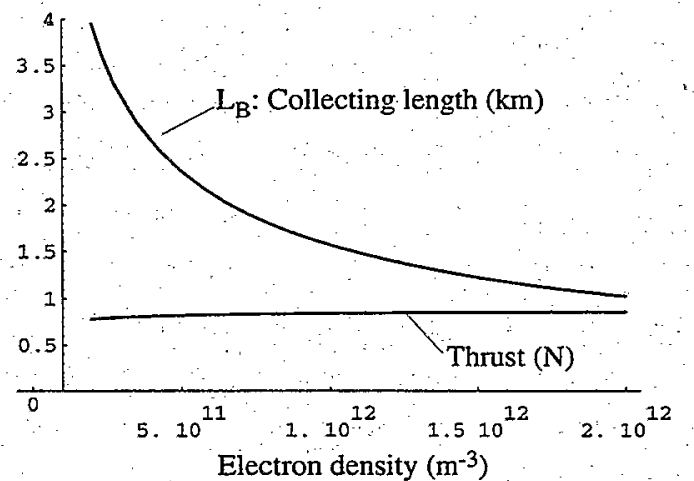

Figure 2-Variation in thrust with electron density for a $10-\mathrm{km}$ tether with a $5-\mathrm{km}-$ long bare segment. Thrust drops only $10 \%$ as density drops by a factor of 10 . The reason is clear: the collecting length has increased from 1 to $4 \mathrm{~km}$.

The EMF is $1200 \mathrm{~V}$; input power $10 \mathrm{~kW}$.

On the whole, the simplicity of the design, the ability to collect high currents, and the reduced sensitivity to density fluctuations, make the baretether concept especially attractive. The OML theory has been substantiated for both quiescent and flowing plasmas in the laboratory, and also in rocket and satellite flights, at moderate voltages $[11,12,18]$.

\section{ProSEDS - The Next Step}

A flight experiment to validate the performance of the bare electrodynamic tether in space and demonstrate its capability to perform thrust is planned by NASA for late year 2000 [9]. The Propulsive Small Expendable Deployer System (ProSEDS) experiment will be placed into a $400-\mathrm{km}$ circular orbit as a secondary payload from a Delta II launch vehicle. The flight-proven Small Expendable
Deployer System (SEDS) will be used to deploy a 5-km predominantly bare aluminum wire attached to $15-\mathrm{km}$ of insulating Spectra tether and $20 \mathrm{~kg}$ endmass. The deployer and endmass mounted on the Delta-II upper stage are shown in Fig. 3.

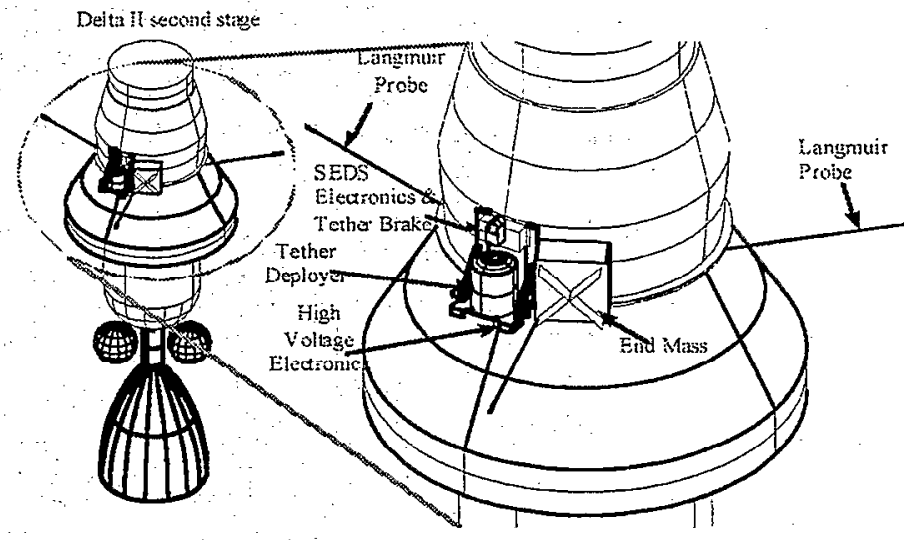

Figure 3-ProSEDS experiment hardware on the Delta-II upper stage.

Once on orbit, the SEDS will reel out the tetherand-endmass system to a total length of $20 \mathrm{~km}$. Upward deployment will set the system to operate in the generator mode, thus producing drag thrust and producing electrical power. The drag thrust provided hy the tether will deorbit the Delta-II upper stage in approximately two to three weeks, versus its nominal 1-year lifetime in a $400-\mathrm{km}$ circular orbit. Approximately $100 \mathrm{~W}$ of electrical power will be extracted from the tether to recharge mission batteries and to allow extended measurements of the system's performance until it re-enters. Predicted performance as a function of average current is shown in Fig. 4.

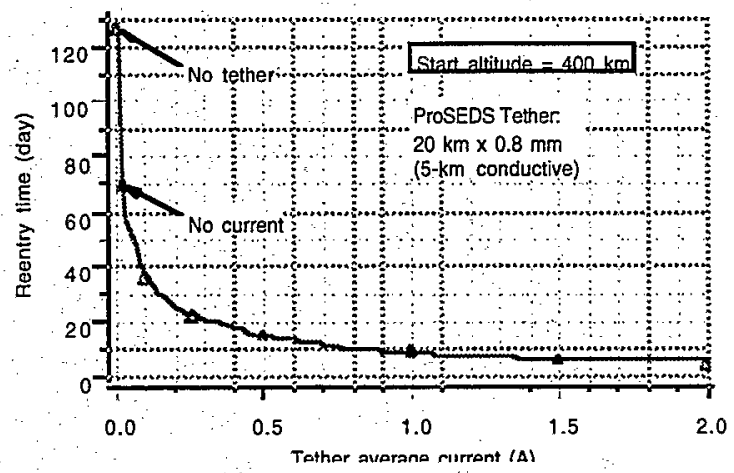

Figure 4 - Predicted ProSEDS performance as a function of average tether current (Courtesy $E$. Lorenzini, SAO). 


\section{Application Examples}

\section{1・Deorbit}

As will be demonstrated by the ProSEDS mission, deorbit of orbiting upper stages and spacecraft at end of mission are feasible: Such applications reduce the amount of propellant that would otherwise be required to accomplish the same task. Further, the relative simplicity of a possible deorbiting ED tether system is appealing when requiring extended lifetime. More detail of such applications are discussed by Hoyt and Forward [13].

\section{2• Orbit Maintenance}

It is possible to consider a relatively short, lowpower ED tether system capable of reboost or drag make-up ${ }^{1}$ and orbit adjustment for repositioning of spacecraft without the requirement for propellant. An important potential candidate is the International Space Station (ISS). In addition, other spacecraft that require long-term operations as low as 250 to $300 \mathrm{~km}$ where drag can be significant may also benefit from ED tether propellantless propulsion.

International Space Station [14] - Outfitting ISS with an ED tether reboost or drag make-up system severs its most critical and constraining dependency on Earth-propellant resupply for reboost or drag make up. The ISS can supply its own electrical power using solar arrays but not its own propellant. It has been estimated that using a $7-\mathrm{km}$ tether and $6 \mathrm{~kW}$ of off-peak electrical power would result in a savings of more than one billion dollars (US \$1 Billion) because of fewer resupply missions.

Even if the planned frequency of resupply flights to the ISS is maintained, with an ED tether the ISS Program has the option to trade kilowatts for increased payload capacity. Resupply vehicles can deliver useful cargo like payloads, replacement parts, and crew supplies rather than propellant. Within the range of 5 to $10 \mathrm{~kW}$, a crude approximation of 1,000 $\mathrm{kg}$ of user payload gained per kilowatt expended per year appears reasonable.

Yet another dimension to propellantless reboost must be considered. Station users have been allocated a minimum of 180 days of microgravity per year. Current planning essentially halts science activity during reboost maneuvers. Low thrust electrodynamic tether drag make-up could be performed over long durations rather than via conventional short-duration, high-thrust propulsive maneuvers. The 0.5 to $0.8 \mathrm{~N}$ thrust provided by an ED tether system more than counteracts atmospheric drag on the ISS. Analysis

1 By "reboost" we mean sufficient thrust levels to accomplish altitude change relatively rapidiy By "drag make-up" we mean low thrust levels sufficient to compensate for atmospheric drag effects. indicates that an ED tether can compensate for the drag while it is occurring, without disrupting the microgravity environment. Fluctuations in the induced voltages from the geomagnetic field and in electron densities will create 'turbulence' through which the ED-tether-driven ISS must fly. How to best compensate for these pockets and maintain microgravity levels remains to be defined. However, the allure of this self-propelled space facility is certainly remarkable and offers potential advantages.

Spacecraft Orbit Maintenance - Other LEO applications besides the ISS can benefit from a propellantless propulsion capability as well. Such a need would be based on a requirement for multiple orbit-modification maneuvers during the spacecraft lifetime (e.g., a remote sensing platform that must be "retargeted"). Another application would be to simply compensate for atmospheric drag to allow the spacecraft to "fly" lower and longer.

Fig. 5 shows an example of using a $2 \mathrm{~km}$-long tether $(1.5-\mathrm{km}$ insulated, $0.5-\mathrm{km}$ bare, $2-\mathrm{mm}$ diameter) that is driven by an electrical power supply providing $150 \mathrm{~W}$ to the tether system to maintain, and even slightly raise, the spacecraft's altitude near $350 \mathrm{~km}$ at $35^{\circ}$ orbit inclination over a week of operation. The spacecraft is assumed to have a mass of $960 \mathrm{~kg}(1000-\mathrm{kg}$ total mass with tether system) and an area of $9 \mathrm{~m}^{2}$. with the tether. Without the tether, there would have been an altitude loss of approximately $5 \mathrm{~km}$ in the same time. To minimize changes in inclination due to ED tether operation, operation was only enabled when the thrust vector was within $25^{\circ}$ of the orbit plane. This resulted in a duty cycle of $-49 \%$. The slight orbit increase over the week time period could be managed by reducing the tether operation further or tightening the thrust-vector control window even further. This analysis assumes no libration or thermal variation due to day-night variation. Also, the electrical energy is assumed to come from an existing on-board power system.

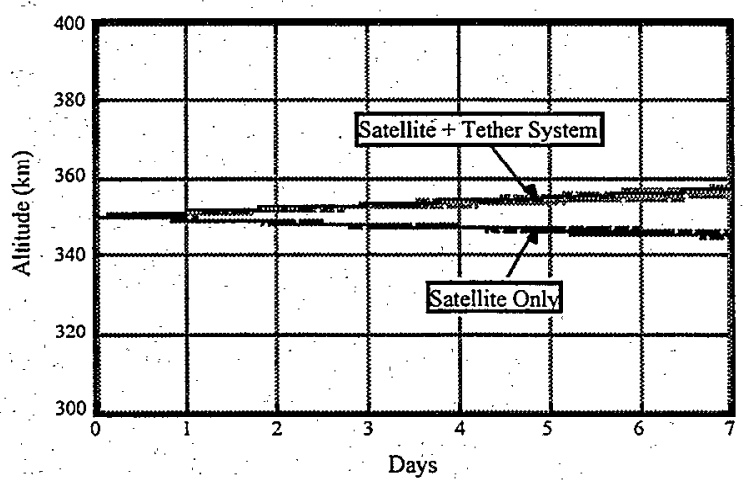

Figure 5- $150-W, 2-\mathrm{km}$ ED-tether orbit maintenance of a $1000 \mathrm{~kg}, 35^{\circ}$ inclination orbit spacecraft system. 


\section{3-Reusable Upper Stage Propulsion}

An ED tether upper stage could also be used as an orbital tug to move payloads in LEO after launch from a reusable launch vehicle (RLV) or other launch vehicle. The tug would rendezvous with the payload and launch vehicle, dock/grapple the payload, and maneuver it to a new orbital altitude or inclination within LEO without the use of boost propellant. The tug could then lower its own orbit to rendezvous with the next payload and repeat the process. Such a system could perform multiple orbital-maneuvering assignments without resupply, substantially lowering recurring costs. The performance of a $10-\mathrm{kW}, 10-\mathrm{km}$ tether system is illustrated in Figures 6 and 7 [10].

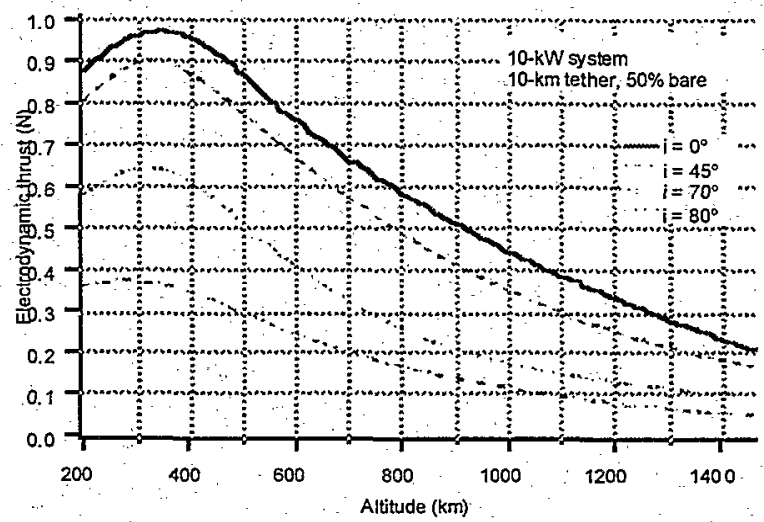

Figure 6-The performance of an electrodynamic tether thruster varies - with altitude in the magnetosphere (where $i$ is the orbital inclination).

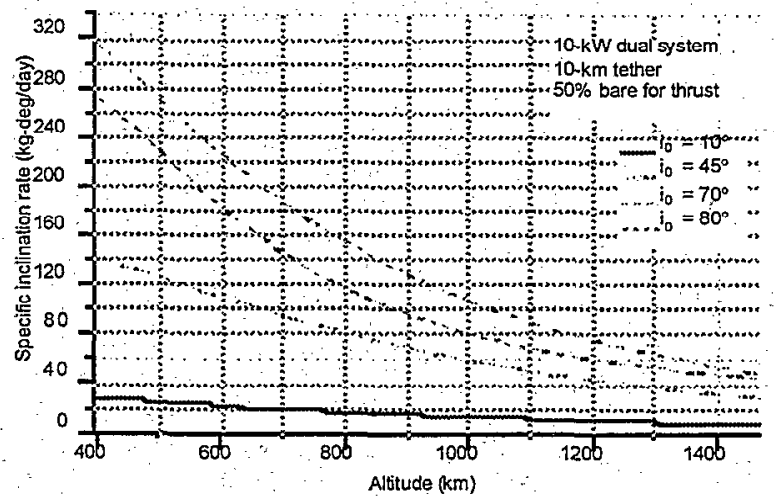

Figure 7-Electrodynamic tethers can be used to change orbital inclination without propellant consumption. To determine the available inclination change for a spacecraft/payload mass, divide the 'specific inclination rate' indicated by the total system mass at a given altitude.

\section{6•The Next Steps for ED Tether Development}

The ProSEDS mission is intended to demonstrate the ability to generate useful tether currents and thrust levels with an emphasis on deorbit thrusting of spent stages. ProSEDS, however, is only a single - but important - step in the process of developing and demonstrating ED tethers for nearterm applications. Important follow-on activities include:

1. Demonstration of orbit-raising and inclination changes and determination of performance over a broader range of altitudes.

2. Replace hollow-cathode electron-emission devices to eliminate the only consumable on present-day ED tethers as well as to simplify systems integration.

3. Develop and demonstrate advanced long-life conducting tethers with improved micrometeoroid and atomic-oxygen resistance and thermal performance.

4. Develop and demonstrate new tether deployers for next-generation tethers.

5. Establish procedures and algorithms for long-term operations of ED tethers including. optimized operations for getting from Point A to Point B and establishing operational limits based on system dynamics.

As a replacement for hollow cathodes (Item 2 above) a recent joint study by the Naval Research Laboratory (NRL) and University of Michigan (UM) [7] identified a new class of field-emitter array for a diverse set of emerging space-based applications. The Field-Emitter Array Cathode (FEAC) offers the potential benefits of low-power consumption, low cost, no consumable requirements, robustness, and high reliability. Space applications that could benefit include: (1) electric propulsion (EP); (2) ED tether propulsion, and (3) spacecraft charge control. Because of their inherent weight, power, and cost advantages, FEACs can provide the enabling technology in some new and significant applications.

FEACs utilize millions of miniature biased emitters attached to a conducting base plate substrate that is imbedded in layers of an insulating metaloxide matrix. A metal grid of gate electrodes are closely placed on top of these layers so as to extract a small current from each emitter by generating a large, localized electric field at the apex of a sharpened structure (field enhancement). An idealized array of emitter tips is shown in Fig. 8. While the number of tips are large, they are cost-effectively built using currently available semiconductor fabrication technology. 


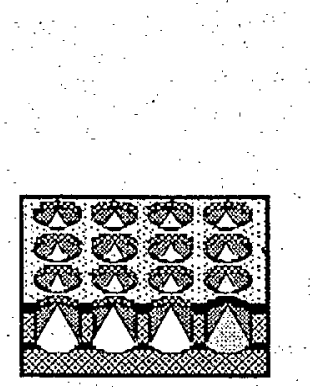

Figure 8-Sketch of a Field Emitter Array Cathode. Typical tip-to-tip separation is on the order of $2-5 \mu \mathrm{m}$.

In recent years, FEAC development has focused on applications such as flat-panel displays and microwave tubes. Their ability to emit electrons without heating or the need for an ionizable gas supply, coupled with recent progress in operating in less than ideal vacuums, ${ }^{2}$ suggest that the time is right to seriously consider their use for space applications as well.

The FEAC space applications generally require devices that: (1) emit on the order of $0.1 \mathrm{~A} / \mathrm{cm}^{2} ;$; operate in the $10^{-5}$ to $10^{-6}$ Torr pressure range (survive after operational exposure to $10^{-3}$ Torr); (3) require bias potentials of $50-100 \mathrm{~V}$ or less; and, (4) have sufficient life for multi-year space operations.

The NRL-UM study found good grounds to believe that each of these requirements can be achieved. What must be done is to bring together the specific set of FEAC fabrication technologies to validate and qualify the technology for operation in the space environment. The study concluded there are three critical FEAC technologies that must be combined. These are: (1) arc suppression at each tip; (2) minimization of bias (gate) potential; and (3) optimizing surface-coatings that lower work-function and withstand contaminating energetic ions, oxygen flux, and undesirable adsorbates.

It appears that FEAC devices that meet these objectives will enable miniaturized fuel-efficient $\mathrm{EP}$ systems such as the Closed-Drift Hall thruster (CDT), which enhance performance of small satellites by reducing spacecraft propellant mass. FEACs will also enable use of "propellantless". ED tether propulsion for efficient spacecraft deorbit, atmospheric drag make-up, and orbit raising in the 250 to 2,000 $\mathrm{km}$ altitude. Additionally, FEACs can provide a simple means to actively control charging of spacecraft with large solar arrays.

\section{Summary}

The ED tether offers a real opportunity for propellantless propulsion in LEO. The impact will be cross-cutting in that potential users will come from both government and commercial applications.

\footnotetext{
2 A summary of the ARPA/NRL/NASA and NRL Programs in RF Vacuum Microelectronics may be found in Jensen et al. [15] and Bandy et al. [16]
}

\section{Acknowledgments}

The authors would like acknowledge the ongoing efforts of the ProSEDS team as it prepares for flight in late 2000 and the support of NASA's Advanced Space Transportation Program for support of ProSEDS and the development of ED tethers as an innovative technology to improve low-cost access in space.

\section{Bibliography}

[1] P. M. Banks, "Review of Electrodynamic Tethers for Space Plasma Science," $J$. Spacecr. Rockets, vol. 26, pp. 234-239, 1989.

[2] M. Dobrowolny, "Electrodynamics of long metallic tethers in the ionospheric plasma," Radio Science, vol. 13, pp. 417-424, 1978.

[3] R. I. Samanta Roy, D. E. Hastings, and E. Ahedo, "Systems analysis of electrodynamic tethers," J. Spacecraft \& Rockets, vol. 29, pp. 415-424, 1992.

[4] M.: Martinez-Sanchez and D. E. Hastings, "A systems study of a $100 \mathrm{~kW}$ electrodynamic tether," The Journal of the Astronautical Sciences, vol. 35, pp. 75-96, 1987.

[5] $\because$ N. H. Stone and C. Bonifazi, "The TSS-1R. mission: Overview and scientific context," Geophys. Res. Ltrs., vol. 25, pp. 409-412, 1998.

[6] D. C. Thompson, C. Bonifazi, B. E. Gilchrist, S. D. Williams, W. J. Raitt, J.P. Lebreton, and W. J. Burke, "The Current-Voltage Characteristics of a Large Probe in Low Earth Orbit: TSS-1R Results," Geophys. Res. Lett., vol. 25, pp. 415-418, 1998.

[7] B. Gilchrist, K. Jensen, J. Severns, and A. Gallimore, "Field Emitter Array Cathodes (FEACs) for Space Applications," presented at 10th NASA/JPL/MSFC/AIAA Advanced Propulsion Research Workshop, Huntsville, AL, 1999.

[8] J. R. Sanmartin, M. Martinez-Sanchez, and E. Ahedo, "Bare wire anodes for electrodynamic tethers," $J$. of Prop. and Power, vol. 9, pp. 353-360, 1993. 
[9] L. Johnson, B. Gilchrist, R. Estes, E. Lorenzini, and J. Ballance, "Propulsive small expendable deployer system (ProSEDS) space experiment," presented at 34th AIAA/ASME/SAE/ASEE Joint Propulsion Conf., Cleveland, $\mathrm{OH}$, AIAA 98-4035, 1998.

[10] L. Johnson, B. Gilchrist, R. Estes, E. Lorenzini, M. Martinez-Sanchez, and J. Sanmartin, "Electrodynamic tether propulsion for spacecraft and upper stages," presented at 1998 JANNAF Propulsion Mceting, Cleveland, OH, 1998.

[11] E. Szuszczewicz and Takacs, Phys. Fluids, vol. 22, pp: 2424, 1979.

[12] Chung and e. al., Probes in Stationary and Flowing Plasmas. New York: Springer, 1975.

[13] R. Hoyt and R. Forward, "Terminator Tether: A low mass device for autonomous deorbit of LEO satellites," presented at 35th AIAA/ASME/SAE/ASEE Joint Propulsion Conf., AIAA-99-2839, Los Angeles, CA, 1999.

[14] L. Johnson and M. Herrmann, "International space station electrodynamic tether reboost study," NASA NASA/TM1998-208538, July 19981998.

[15] K. L. Jensen, R. H. Abrams, and R. K. Parker, "Field emitter array development for high frequency applications," J. Vac. Sci. Technol., vol. B16, pp. 749, 1998.

[16] S. G. Bandy, M. C. Green, C. A. Spindt, M. A. Hollis, W. D. Palmer, B. Goplen, and E. G. Wintucky, presented at Tech. Digest of the 11th Int'1 Vacuum Microelectronics Conference, Asheville, NC, 1998.

[17] Laframboise, U. of Toronto Inst. for Space Res. Report 100, 1966.

[18] Mercure, U. of Toronto Inst. for Aerospace Studies Report No. 202, 1976. 